\title{
Computed tomography-based visual assessment of chronic obstructive pulmonary disease: comparison with pulmonary function test and quantitative computed tomography
}

\author{
Han Sol Kang ${ }^{1} \wedge$, So Hyeon Bak ${ }^{1}$, Ha Yeun $\mathrm{Oh}^{1}{ }^{\wedge}$, Myoung-Nam $\mathrm{Lim}^{2} \wedge$, Yoon Ki Cha ${ }^{3} \wedge$, \\ Hyun Jung Yoon $^{4} \wedge$, Woo Jin Kim $^{5} \wedge$
}

${ }^{1}$ Department of Radiology, Kangwon National University Hospital, Kangwon National University School of Medicine, Chuncheon, Republic of Korea; ${ }^{2}$ Biomedical Research Institute, Kangwon National University Hospital, Chuncheon, Republic of Korea; ${ }^{3}$ Department of Radiology and Center for Imaging Science, Samsung Medical Center, Sungkyunkwan University School of Medicine, Seoul, Republic of Korea; ${ }^{4}$ Department of Radiology, Veterans Health Service Medical Center, Seoul, Republic of Korea; ${ }^{5}$ Department of Internal Medicine and Environmental Health Center, School of Medicine, Kangwon National University, Chuncheon, Republic of Korea

Contributions: (I) Conception and design: SH Bak; (II) Administrative support: All authors; (III) Provision of study material or patients: All authors; (IV) Collection and assembly of data: HS Kang, SH Bak, WJ Kim; (V) Data analysis and interpretation: All authors; (VI) Manuscript writing: All authors; (VII) Final approval of manuscript: All authors.

Correspondence to: So Hyeon Bak. Department of Radiology, Kangwon National University Hospital, Kangwon National University School of Medicine, 1 Kangwondaehak-gil, Chuncheon, Gangwon-do 24341, Republic of Korea. Email: arsgnm17@gmail.com.

\begin{abstract}
Background: Chronic obstructive pulmonary disease (COPD) has variable subtypes involving mixture of large airway inflammation, small airway disease, and emphysema. This study evaluated the relationship between visually assessed computed tomography (CT) subtypes and clinical/imaging characteristics.

Methods: In total, 452 participants were enrolled in this study between 2012 and 2017. Seven subtypes were defined by visual evaluation of CT images using Fleischner Society classification: normal, paraseptal emphysema (PSE), bronchial disease, and centrilobular emphysema (trace, mild, moderate and confluent/ advanced destructive). The differences in several variables, including clinical, laboratory, spirometric, and quantitative CT features among CT-based visual subtypes, were compared using the chi-square tests and one-way analysis of variance.
\end{abstract}

Results: Subjects who had PSE had better forced expiratory volume in 1 second (FEV1) $(\mathrm{P}=0.03)$ percentage and higher lung density $(\mathrm{P}<0.05)$ than those with moderate to confluent/advanced destructive centrilobular emphysema. As the visual grade of centrilobular emphysema worsened, pulmonary function declined and modified Medical Research Council, COPD assessment test (CAT) score, and quantitative assessment (emphysema index and air trapping) increased. The bronchial subtype was associated with higher body mass index (BMI), better lung function and higher lung density. Participants with trace emphysema showed a rapid increase in functional small airway disease

Conclusions: Classifying subtypes using visual CT imaging features can reflect heterogeneity and pathological processes of COPD.

Keywords: Chronic obstructive pulmonary disease (COPD); computed tomography (CT); phenotype; quantification

^ ORCID: Han Sol Kang, 0000-0002-3238-4438; So Hyeon Bak, 0000-0003-1039-7016; Ha Yeun Oh, 0000-0001-8591-6947; MyoungNam Lim, 0000-0003-3562-0667; Yoon Ki Cha, 0000-0002-5960-0719; Hyun Jung Yoon, 0000-0002-8909-1185; Woo Jin Kim, 0000-00032927-370X. 
Submitted Oct 09, 2020. Accepted for publication Jan 13, 2021.

doi: $10.21037 /$ jtd-20-3041

View this article at: http://dx.doi.org/10.21037/jtd-20-3041

\section{Introduction}

Chronic obstructive pulmonary disease (COPD) is characterized by nonreversible airflow limitation caused by the inhalation of irritants such as tobacco smoke (1). Approximately $15-20 \%$ of smokers are likely to develop COPD, and there is substantial heterogeneity in the clinical manifestation, physiological characteristics, imaging characteristics, disease progression, and prognosis of the disease $(1,2)$. COPD is routinely diagnosed based on pulmonary function test (PFT) results, respiratory symptoms, and history of exposure (3). Nevertheless, the technique has several limitations. PFT results are difficult to evaluate in patients with respiratory problem, and tests tends to be relatively insensitive at the early stage of the disease, and cannot distinguish the subtypes of COPD, including emphysema, small airway disease, and bronchial inflammation $(2,4)$.

Computed tomography (CT) is a useful imaging modality for evaluating structural abnormalities and for classifying the COPD subtype. Quantitative CT evaluation is useful for assessing the severity of emphysema, airway wall thickening, and small airway disease (5). Emphysema quantification by means of CT correlated well with histopathology $(6,7)$. However, CT densitometry has limited ability to provide information about the emphysema phenotype. Recently, visual categorization based on CT was proposed by the Fleischner Society, and emphysema has been divided into centrilobular emphysema, paraseptal emphysema (PSE), and panlobular emphysema (8). A study reported that the visual presence and severity of emphysema was an independent predictor of mortality (9). However, the study analyzed only centrilobular emphysema, and did not analyze other COPD phenotypes, such as PSE and bronchial disease. Recently, Park et al. (2) reported that the phenotypes of COPD could classified by combining visual and quantitative CT imaging features, presenting a useful tool for characterizing the heterogeneity of COPD. Nevertheless, quantitative CT evaluation of COPD is not yet widely used in clinical practice. Therefore, we here classified COPD phenotypes by means of visual characterization only, in order to evaluate the relationship of visually assessed CT subtypes with clinical presentation and quantitative CT characteristics.

We present the following article in accordance with the STROBE reporting checklist (available at http://dx.doi. org/10.21037/jtd-20-3041).

\section{Methods}

\section{Study population}

Five-hundred-and-four participants from the Chronic Obstructive Pulmonary Disease in Dusty Areas (CODA) cohort, a Korean study that observed the clinical outcomes of COPD in Korean patients residing near cement plants, were initially selected. The details of this cohort had been described previously (10). All enrolled participants underwent medical interview, physical examination, spirometry, and chest CT scan. We excluded 52 participants with quantification error $(n=8)$, lobectomy state $(n=4)$, and severe lung parenchymal distortion due to pneumoconiosis, bronchiectasis, and pulmonary tuberculosis $(n=30)$, and accompanying lung disease $(n=10)$ that could affect the quantitative CT measurement. Finally, 452 participants were enrolled (Figure 1). The study was conducted in accordance with the Declaration of Helsinki (as revised in 2013). This study was approved by the institutional review board of our hospital (approval \#2012-06-007), and all participants provided written informed consent.

\section{Clinical parameters and pulmonary function}

The interview questionnaire included questions on demographic data, medical history, environmental exposure, and respiratory symptoms. Dyspnea was assessed using the modified Medical Research Council, and healthrelated quality of life was assessed using the patientreported COPD assessment test (CAT). The peripheral levels of white blood cells (WBCs), neutrophil, eosinophils, interleukin (IL)-6, IL-8, and C-reactive protein were used as markers of inflammation.

PFTs were performed using the Easy One Kit (NDD Medizintechnik AG, Zurich, Switzerland), before and after inhalation of $400 \mu \mathrm{g}$ salbutamol. The spirometric airflow limitation is defined as a postbronchodilator ratio of forced 


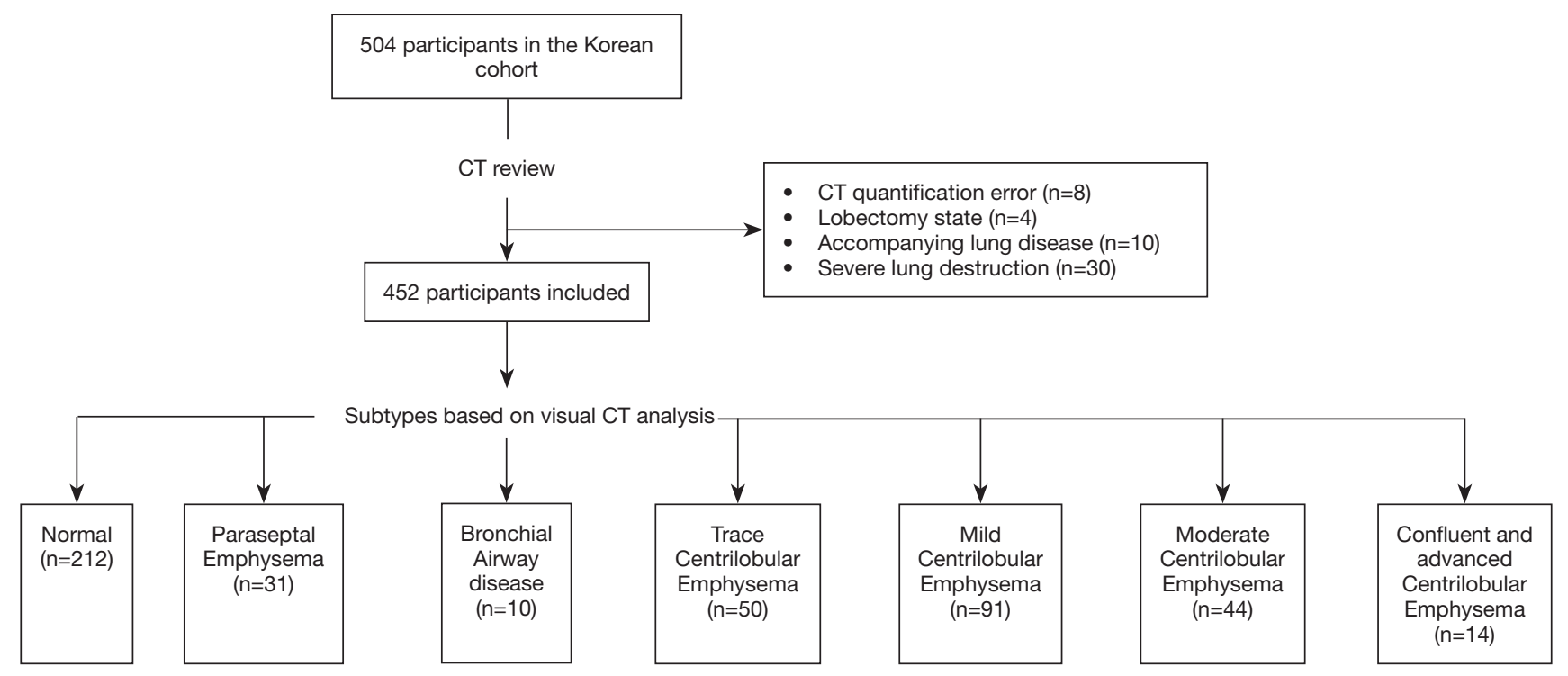

Figure 1 Flowchart of the study participants.

expiratory volume in 1 second (FEV1) to forced vital capacity (FVC) of $<0.7$. The severity of airflow limitation was classified as described by the 2017 Global Initiative for Chronic Obstructive Lung Disease (GOLD) grading system as grade $1(\geq 80 \%)$, grade $2(50-79 \%)$, grade $3(30-49 \%)$, or grade $4(<30 \%)(11)$. GOLD grade 0 was defined as normal spirometry, and the group with normal FEV1/FVC and $\mathrm{FEV} 1<80 \%$ predicted was defined as having preserved ratio impaired spirometry (PRISm) (3). Bronchodilator responsiveness (BDR) was defined as an increase of at least $12 \%$ or $200 \mathrm{~mL}$ in FEV1 or FVC after salbutamol administration (12).

\section{CT acquisition}

Participants underwent thin-section, chest CT at full inspiration and expiration in the supine position. All chest CT examinations were performed using a Somatom Definition dual-source CT scanner (Siemens Healthcare, Forchheim, Germany) with the following parameters: $140 \mathrm{kVp}, 100 \mathrm{~mA}, 09-1$ beam pitch and $0.6 \mathrm{~mm}$ slice thickness. CT images were reconstructed with B30f.

\section{Visual analysis and subtypes based on CT images}

CT scans were visually assessed on the basis of the Fleischner Society classification system (8). Centrilobular emphysema was graded as trace (ca. $0.5 \%)$, mild $(0.5-5 \%)$, moderate $(>5 \%)$, confluent (coalescent centrilobular or lobular lucencies) and advanced destructive emphysema (panlobular lucencies with hyperexpansion and distortion). Substantial PSE was defined as large cyst-like lucencies located near the pleura and interlobar fissure. Visual assessment of CT was independently interpreted by 2 radiologists (with 3 and 11 years of experience in thoracic CT scan interpretation) who were unaware of clinical data. Different interpretations were resolved by consensus.

Participants were categorized into 7 subtypes based on visual assessment of CT images: (I) normal (subjects with no visual emphysema and bronchial wall thickening), (II) PSE (subjects with substantial PSE regardless of other imaging characteristics), (III) bronchial airway disease (subjects with bronchial wall thickening and absent or trace emphysema or mild PSE), (IV) trace centrilobular emphysema, (V) mild centrilobular emphysema, (VI) moderate centrilobular emphysema, and (VII) confluent and advanced destructive emphysema (Figure 2) (2).

\section{Quantitative analysis based on CT image}

Lung segmentation and image co-registration of inspiratory and expiratory CT were performed using an Aview ${ }^{\circledR}$ system (Coreline Soft Inc., Seoul, South Korea). Parametric response mapping (PRM) analysis was classified into $\mathrm{PRM}^{\mathrm{fSAD}}(\geq-950 \mathrm{HU}$ at inspiration and $<-856 \mathrm{HU}$ at expiration), or $\mathrm{PRM}^{\mathrm{emph}}(<-950 \mathrm{HU}$ at inspiration and 

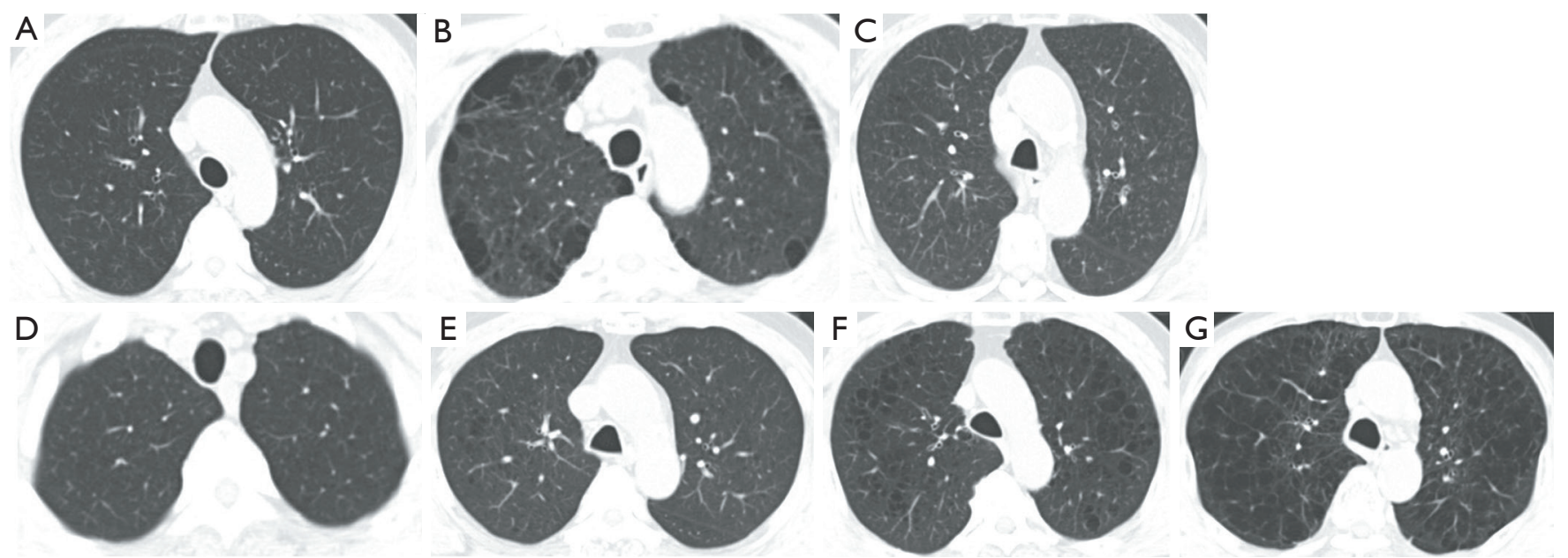

Figure 2 Classification of 7 computed tomography (CT) subtypes. (A) Normal. (B) Paraseptal emphysema; CT scan shows numerous low attenuation areas in the subpleural regions. (C) Bronchial disease; CT scan shows diffuse bronchial wall thickening without emphysema. (D) Trace centrilobular emphysema; CT scan shows scattered centrilobular low attenuated lesions involving about $0.5-5 \%$ of the upper lung zone. (E) Mild centrilobular emphysema; CT scans shows well-defined low attenuation lesions involving about $0.5-5 \%$ of the upper lung zone. (F) Moderate centrilobular emphysema; CT scans shows well-defined low attenuated lesions involving more than $5 \%$ of the upper lung zone. (G) Confluent and advanced destructive emphysema; CT scans shows hyperexpanded secondary pulmonary lobules and multiple hyperlucencies in both lungs.

$<-856 \mathrm{HU}$ at expiration, Figure 3) (13). PRM data were represented as a percentage of the total lung volume. Lung attenuation at the $15^{\text {th }}$ percentile (Perc 15) was defined as CT attenuation at the 15 th percentile of the lung CT histogram (14). Pi10 was defined as the airway wall area standardized to an internal airway perimeter of $10 \mathrm{~mm}$.

\section{Statistical analysis}

Parametric data are expressed as mean \pm standard deviation (SD), whereas nonparametric data are expressed as numbers and percentages. The chi-square test and one-way analysis of variance were used to determine differences in clinical characteristics and quantitative CT variables among the subtypes. $\mathrm{P}$ values $<0.05$ were considered statistically significant. Data analysis was performed using SAS version 9.3 (SAS Institute Inc., Cary, NC, USA).

\section{Results}

\section{Study population}

For the 452 participants, the mean age at enrollment was $72.4 \pm 7.4$ years, and 327 of these participants $(72.4 \%)$ were male. Two-hundred-and-eighty-five participants (63.5\%) were current or former smokers at enrollment, while 3 participants had no recorded smoking history. With the subjects group divided into GOLD grades, 142 participants (31.4\%) were GOLD grade 0, 20 participants (4.4\%) were PRISm, 148 participants (32.7\%) were GOLD grade 1, 119 participants $(26.3 \%)$ were GOLD grade 2, 21 participants (4.7\%) were GOLD grade 3 and only 2 participants $(0.5 \%)$ were GOLD grade 4 . Detailed characteristics of the participants are shown in Table 1.

\section{Overall comparison of subtypes}

Subjects were divided into 7 subtypes. There were 212 (46.9\%) subjects with no abnormality in visual CT analysis. Thirty-one (6.9\%) subjects had PSE type and 10 (2.2\%) had bronchial type. One-hundred-and-ninety-nine (44.0\%) subjects had centrilobular emphysema on visual CT analysis and trace, mild and moderate centrilobular emphysema (11.1\%, $20.1 \%$ and $9.7 \%$ each respectively) were predominant type among the 7 subtypes (Table 2).

Body mass index (BMI) lowered statistically significantly as the degree of emphysema, based on CT findings, worsened. The bronchial group tended to have a higher BMI $(24.2 \pm 3.2)$ than the emphysema groups, except for the normal group. The CAT score was highest in subjects 

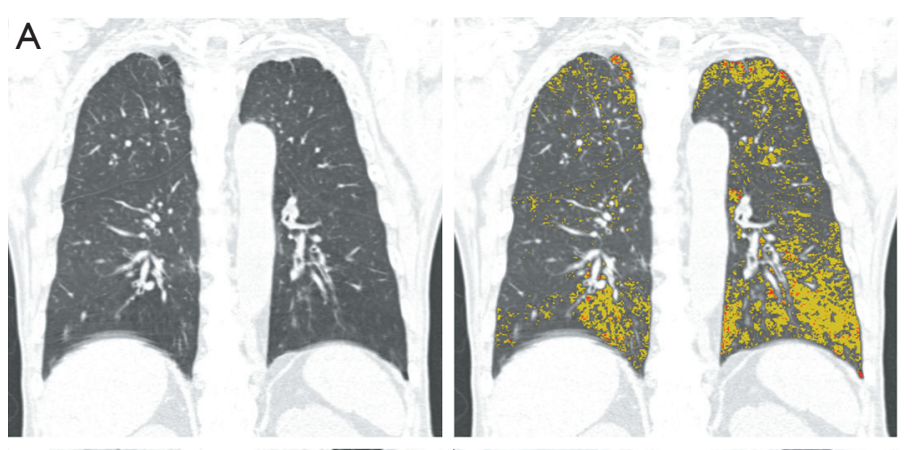

B
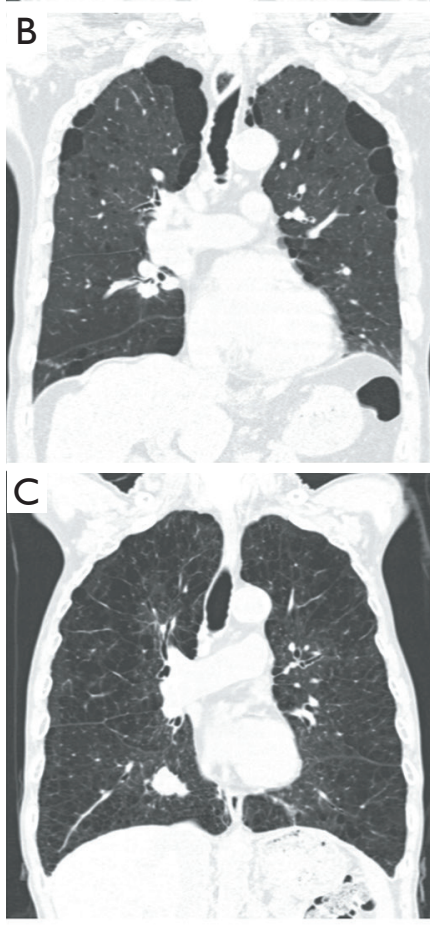

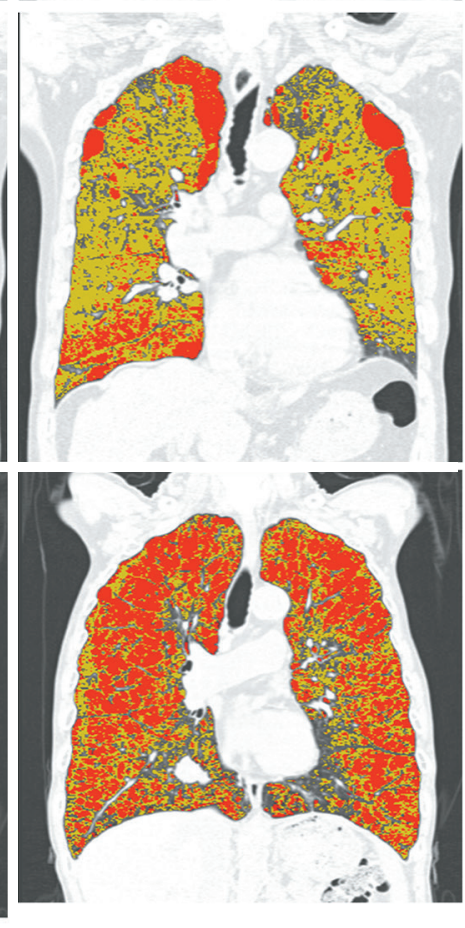

Normal

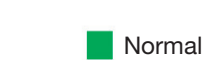

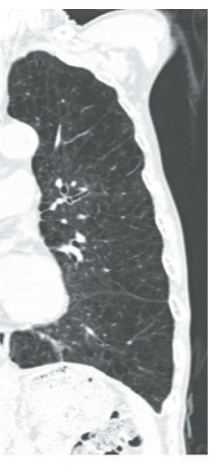

Figure 3 Parametric response mapping in 3 different patients. Normal lung tissue is denoted in green $\left(\mathrm{PRM}^{\mathrm{normal}}\right)$, functional small airway disease in yellow $\left(\mathrm{PRM}^{\mathrm{fSAD}}\right)$, and emphysematous lung in red $\left(\mathrm{PRM}^{\mathrm{emphy}}\right)$. (A) Image of a 56-year-old male with COPD GOLD grade 2. CT shows significant bronchial wall thickening without emphysema. CT subtype was assigned as bronchial disease subtype. PRM ${ }^{\text {emphy }}$ was $0.7 \%$ and $\mathrm{PRM}^{\mathrm{fSAD}}$ was $19.2 \%$. Pi-10 was $5.5 \mathrm{~mm}$. (B) Image of a 78-year-old male with COPD GOLD grade 4 . CT shows paraseptal emphysema in both upper lungs. CT subtypes was classified paraseptal emphysema. PRM ${ }^{\text {emphy }}$ was $18.2 \%$ and $\mathrm{PRM}{ }^{\mathrm{fSAD}}$ was $50.4 \%$. (C) Image of a 78-year-old male with COPD GOLD grade 2. CT show diffuse lobular lucencies with hyperexpansion in both lungs. CT subtype was classified as advanced destructive emphysema. $\mathrm{PRM}^{\text {emphy }}$ was $38.5 \%$ and $\mathrm{PRM}^{\mathrm{fSAD}}$ was $30.0 \%$. CT, computed tomography; COPD, chronic obstructive pulmonary disease; GOLD, Global Initiative for Chronic Obstructive Lung Disease.

with PSE (20.5 \pm 9.6$)$. Although there was no statistically significant difference, neutrophil counts were the highest in subjects with PSE. In subjects with PSE, IL-6 was higher than in other subtypes $(\mathrm{P}=0.004)$. Pulmonary function lowered as the degree of emphysema worsened.

The Pi-10 was greatest in the bronchial disease subtype.
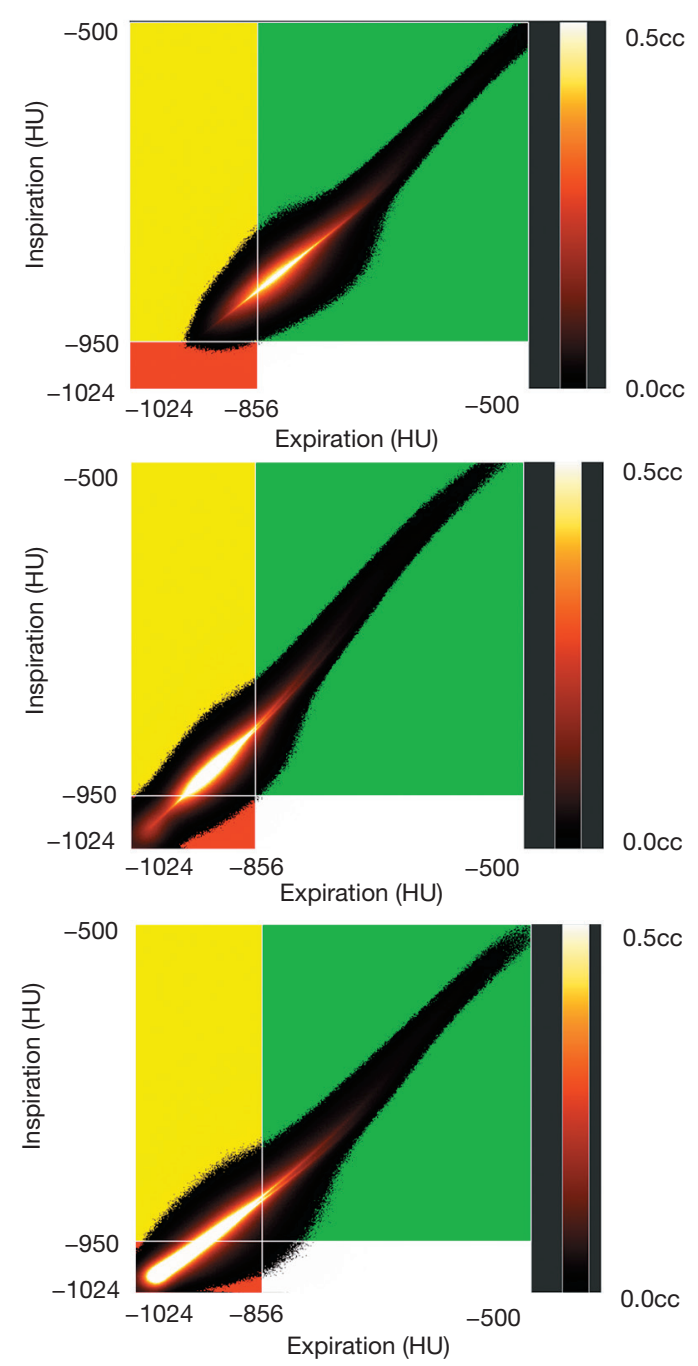

Emphysema $\square$ Uncategorized 
Table 1 Characteristics of 452 participants

\begin{tabular}{|c|c|}
\hline Characteristics & Value \\
\hline Age & $72.4 \pm 7.4$ \\
\hline Male* & $327(72.4)$ \\
\hline BMI & $23.6 \pm 3.2$ \\
\hline \multicolumn{2}{|l|}{ Smoking status* } \\
\hline Current & $98(21.8)$ \\
\hline Former & $187(41.7)$ \\
\hline None & $164(36.5)$ \\
\hline Pack-years & $17.5 \pm 23.6$ \\
\hline $\mathrm{mMRC}$ & $1.36 \pm 1.1$ \\
\hline CAT score & $16.1 \pm 5.6$ \\
\hline WBC $\left(\times 10^{3} / \mu \mathrm{L}\right)$ & $6.8 \pm 1.9$ \\
\hline Neutrophil (\%) & $58.5 \pm 9.5$ \\
\hline Eosinophil (\%) & $2.7 \pm 2.8$ \\
\hline $\mathrm{CRP}(\mathrm{mg} / \mathrm{dL})$ & $0.3 \pm 0.6$ \\
\hline IL-6 (pg/mL) & $2.5 \pm 3.5$ \\
\hline IL-8 (pg/mL) & $17.7 \pm 21.3$ \\
\hline \multicolumn{2}{|l|}{ GOLD grades* } \\
\hline 0 & $142(31.4)$ \\
\hline PRISm & $20(4.4)$ \\
\hline 1 & $148(32.7)$ \\
\hline 2 & $119(26.3)$ \\
\hline 3 & $21(4.7)$ \\
\hline 4 & $2(0.5)$ \\
\hline \multicolumn{2}{|c|}{ Pulmonary function test } \\
\hline FVC (L) & $3.0 \pm 0.8$ \\
\hline FVC (\%) & $97.9 \pm 18.7$ \\
\hline FEV1 (L) & $1.96 \pm 0.6$ \\
\hline FEV1 (\%) & $87.5 \pm 22.3$ \\
\hline FEV1/FVC & $65.2 \pm 11.5$ \\
\hline $\mathrm{BDR}^{\star}(\%)$ & $70(15.5)$ \\
\hline
\end{tabular}

Data are shown as mean \pm standard deviation unless indicated otherwise. *Data are number ad data in parentheses are percentages. BDR, bronchodilator responsiveness; BMI, body mass index; CAT, chronic obstructive pulmonary disease assessment test; CRP, C-reactive protein; FEV1, forced expiratory volume in $1 \mathrm{~s}$; FVC, forced vital capacity; GOLD, Global Initiative for Chronic Obstructive Lung Disease; mMRC, modified Medical Research Council. in the mild emphysema group as compared to the trace emphysema group. The differences among the subtypes are shown in Table 2.

\section{PSE compared with moderate and severe emphysema}

Participants with confluent and advanced destructive emphysema had the lowest lung function and highest emphysema $(\mathrm{P}<0.05)$. Compared with participants with moderate centrilobular emphysema, participants with PSE had lower lung function and Perc 15, and higher PRM ${ }^{\text {emph }}$. Although there were no statistically significant differences, functional small airway disease was least common in participants with PSE (Table 3).

\section{Discussion}

In this study, we demonstrate that COPD phenotypes could be evaluated by using only visual assessment of CT images. We found that pulmonary function and CT quantitative characteristics of PSE were intermediate to those of moderate emphysema and confluent advanced destructive emphysema. Participants with PSE had the highest CAT score, neutrophil count, and IL-6 levels among the subtypes. In addition, participants with trace emphysema showed a higher degree in functional small airway disease among the emphysema subtypes. Pulmonary function and quantitative features worsened with the visual grade of emphysema.

The diagnosis, severity evaluation and longitudinal monitoring of COPD usually depend on PFTs, including FEV1/FVC and FEV1. However, PFT can underestimate lung function in younger patients and overestimate lung function in older (15). A previous study found that FEV1 did not significantly reflect the progression of emphysema and air trapping detected by CT (less than $10 \%$ of emphysema and up to $50 \%$ for air trapping) (16). In addition, PFT provides a global evaluation of lung damage, rather than regional information (17). CT could be used to evaluate the extent, subtype, distribution and progression of COPD (15). Quantitative CT analysis provides global and regional information regarding emphysema, air trapping, and airways (8), and in patients with COPD, quantitative CT analysis generally correlated with pathology-based quantification of emphysema and the results of PFT (17). A study showed that emphysema associated with the FEV1/ FVC, Pi10 did with the FEV1, and the ratio of mean lung 
density at expiration and inspiration (E/I-ratioMLD) did with the residual volume (18). PRM for COPD quantification is a voxel-based image analysis technique between inspiration and expiration scans after image registration (19). Pompe et al. showed that $\mathrm{PRM}^{\mathrm{emph}}$ and $\mathrm{PRM}^{\mathrm{fSAD}}$ strongly associated with presence and severity of COPD (20). In addition, the baseline model (age, BMI, smoking status and packyears) with PRM had higher diagnostic value compared with other CT-derived biomarkers (Pi10, Perc 15, E/ I-ratio $_{\text {MLD }}$ ) (20). Therefore, in our study, PRM $^{\text {emph }}$ and $\mathrm{PRM}^{\mathrm{fSAD}}$ were used as the COPD quantification biomarkers. However, the heterogeneous nature of COPD cannot be explained by quantitative CT features only (4). In addition to quantitative analysis, visual evaluation of CT images provides information on patterns of altered lung structure and distinct phenotypes in COPD that cannot currently be identified by quantitative CT (8). A previous study showed that visual assessment of COPD could provide reproducible, physiologically significant information (21).

Emphysema is classified into centrilobular emphysema, PSE, and panlobular emphysema. Centrilobular emphysema shows destruction of the center of the lung lobule, whereas PSE shows destruction of peripheral lung lobule located near the pleural surface and in the interlobular fissures (8).
The clinical manifestation and pathologic features differ between PSE and centrilobular emphysema. Compared with subjects with PSE, subjects with centrilobular emphysema tended to have more dyspnea, reduced exercise capacity, and impaired lung function $(2,22)$. The number of terminal bronchioles are relatively preserved in PSEdominant regions compared to centrilobular emphysema dominant regions in patients with COPD, which implies that patients with the PSE had better physiological and functional characteristics (23). In our study, participants with PSE had the highest CAT score, particularly regarding items on sputum and dyspnea when climbing stairs. Our study classified PSE subtypes in subjects with substantial PSE regardless of other characteristics; most subjects with moderate to advanced destructive emphysema were included in the PSE subtype group. Therefore, this group may have slightly different physiological and functional characteristics to a pure PSE. As in other studies, compared with participants with confluent and advanced destructive emphysema, participants with PSE had better pulmonary function, and higher lung density.

COPD is associated with chronic inflammation, which is characterized by innate and adaptive immune responses (24). The infiltration of macrophages, $\mathrm{CD}^{+}-, \mathrm{CD}^{+}-$and

Table 2 Clinical and imaging characteristics in 7 computed tomography imaging subtypes

\begin{tabular}{|c|c|c|c|c|c|c|c|c|}
\hline Characteristics & Normal & PSE & Bronchial & Trace & Mild & Moderate & $\begin{array}{c}\text { Confluent and } \\
\text { advanced destructive }\end{array}$ & $P$ value \\
\hline Number of subjects & 212 (46.9) & $31(6.9)$ & $10(2.2)$ & $50(11.1)$ & $91(20.1)$ & $44(9.7)$ & $14(3.1)$ & \\
\hline Male* & $119(56.1)$ & $31(100.0)$ & $5(50.0)$ & $36(72.0)$ & $80(87.9)$ & $42(95.5)$ & $14(100.0)$ & $<0.001$ \\
\hline BMI & $24.6 \pm 3.0$ & $22.5 \pm 3.6$ & $24.2 \pm 3.2$ & $24.3 \pm 3.2$ & $22.5 \pm 2.8$ & $21.8 \pm 3.1$ & $20.4 \pm 2.3$ & $<0.001$ \\
\hline Current & $26(12.3)$ & $10(32.3)$ & $3(30.0)$ & $8(16.0)$ & $27(29.7)$ & $20(48.8)$ & $4(28.6)$ & \\
\hline Former & $63(29.7)$ & $20(64.5)$ & $2(20.0)$ & $22(44.0)$ & $51(56.0)$ & $19(46.3)$ & $10(71.4)$ & \\
\hline None & $123(58.0)$ & $1(3.2)$ & $5(50.0)$ & $20(40.0)$ & $13(14.3)$ & $2(4.9)$ & $0(0.0)$ & \\
\hline Neutrophil (\%) & $57.3 \pm 10.1$ & $62.0 \pm 8.1$ & $60.0 \pm 10.4$ & $57.2 \pm 9.8$ & $60.0 \pm 8.7$ & $59.0 \pm 8.4$ & $59.3 \pm 7.9$ & 0.087 \\
\hline Eosinophil (\%) & $2.6 \pm 2.3$ & $2.7 \pm 4.5$ & $2.4 \pm 1.5$ & $3.3 \pm 4.8$ & $2.4 \pm 2.1$ & $2.8 \pm 2.0$ & $2.0 \pm 1.5$ & 0.717 \\
\hline $\mathrm{CRP}(\mathrm{mg} / \mathrm{dL})$ & $0.3 \pm 0.7$ & $0.4 \pm 0.9$ & $0.2 \pm 0.3$ & $0.2 \pm 0.2$ & $0.2 \pm 0.5$ & $0.3 \pm 0.4$ & $0.4 \pm 0.7$ & 0.786 \\
\hline
\end{tabular}

Table 2 (continued) 
Table 2 (continued)

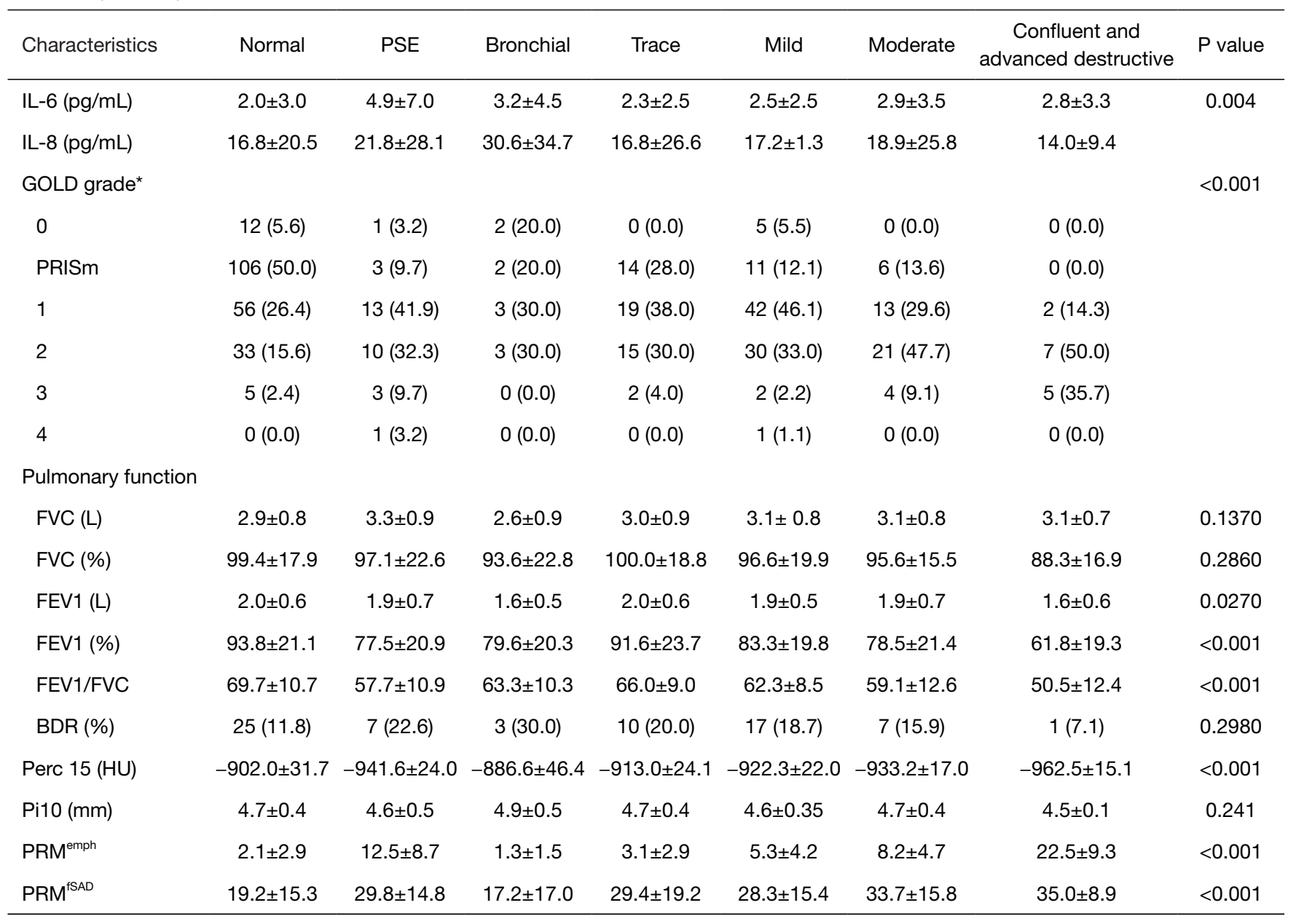

Data are shown as mean \pm standard deviation unless indicated otherwise. *Data are number ad data in parentheses are percentages. BDR, bronchodilator responsiveness; BMI, body mass index; CAT, chronic obstructive pulmonary disease assessment test; CRP, C-reactive protein; FEV1, forced expiratory volume in $1 \mathrm{~s}$; FVC, forced vital capacity; GOLD, Global Initiative for Chronic Obstructive Lung Disease; mMRC, modified Medical Research Council; Perc 15, lung attenuation at 15th percentile; PRM, parametric response mapping; PSE, paraseptal emphysema.

B-cell lymphocytes were associated with the centrilobular phenotype of emphysematous destruction in COPD (25). On the other hand, histological evaluation showed that neutrophil infiltration is increased in PSE regions (26). In our study, the PSE subtype showed the highest neutrophil count among the subtypes. Neutrophils secrete neutrophil elastase, cathepsin G, and proteinase-3, which lead to alveolar destruction (24). Neutrophilia and sputum neutrophil count correlated with the rate of decline in lung function and peripheral airway dysfunction on CT (27). Tanabe et al. (23) explained that toxic gas spreads to the periphery of the lobule leading to PSE, which leads to an increase in neutrophils. In addition to neutrophils, pro-inflammatory cytokines also play an important role in the pathogenesis of COPD (28). In the present study, the PSE subtype showed significantly higher IL-6 levels; IL-6 increases alveolar cell apoptosis and promotes pulmonary emphysema (29).

Our study showed that functional small airway disease in the trace emphysema subtype had higher degree than in the mild emphysema subtype. Airflow limitation occurred in small airways less than $2 \mathrm{~mm}$ in diameter. Since these small airways are beyond the current CT imaging resolution, it is assumed that air trapping is caused by small airway disease (30). Several studies have shown that the narrowing and disappearance of small conducting airways precede emphysematous destruction $(31,32)$. PRM is significant 
Table 3 Clinical and imaging characteristics in paraseptal emphysema, moderate centrilobular emphysema and severe emphysema subtypes

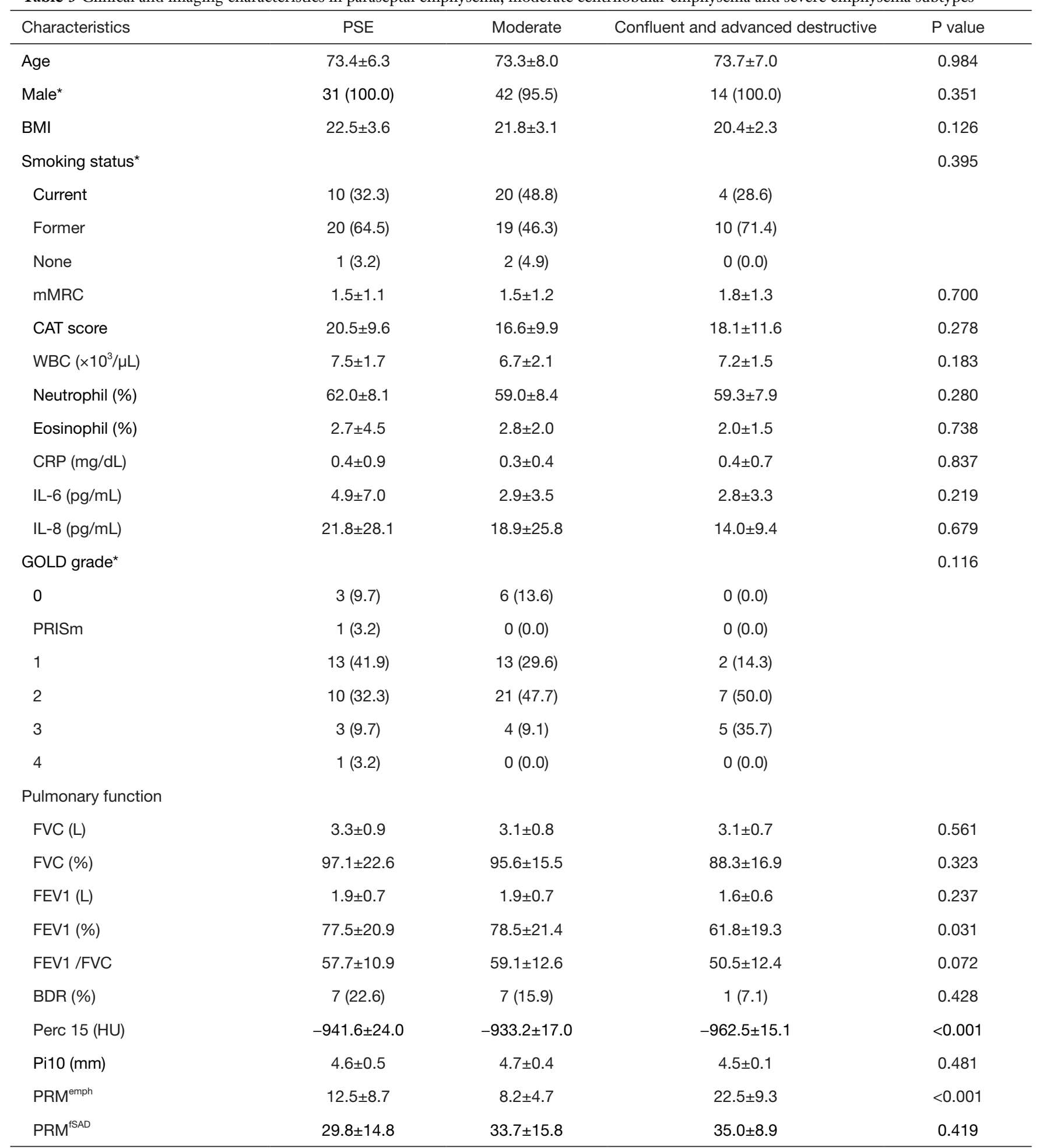

Data are shown as mean \pm standard deviation unless indicated otherwise. *Data are number ad data in parentheses are percentages. BDR, bronchodilator responsiveness; BMI, body mass index; CAT, chronic obstructive pulmonary disease assessment test; CRP, C-reactive protein; FEV1, forced expiratory volume in $1 \mathrm{~s}$; FVC, forced vital capacity; GOLD, Global Initiative for Chronic Obstructive Lung Disease; mMRC, modified Medical Research Council; Perc 15, lung attenuation at 15th percentile; PRM, parametric response mapping; PSE, paraseptal emphysema. 
diagnostic value of CT quantification because of its ability to differentiation of emphysema from non-emphysematous air trapping (function small airway disease, $\mathrm{PRM}^{\mathrm{fSAD}}$ ) within lung parenhcyma (19). Also, several studies have shown that PRM are valuable CT derived biomarker assessment of small airway disease and COPD regardless of subtypes of COPD $(19,20)$. Our study using PRM demonstrate that functional small airway disease occurs in the trace emphysema subtype, in the early stage of the disease, and that emphysema increased subsequently, supporting previous studies that indicated that small airway disease is a precursor to emphysema development (30).

Our study showed that participants with bronchial disease tended to have a higher BMI, airway thickness, and lung density, and better lung function. BMI is associated with the severity of emphysema: as BMI lowered, emphysema worsened (33). In contrast, BMI and airway thickness were positively correlated (34). In our study, the bronchial subtype had the highest BMI among the groups, except for the normal group. Due to airway inflammation, the bronchial subtype had higher value of inflammatory markers, including WBC and neutrophil count, and IL-6 and IL-8 levels.

This study had several limitations. First, the number of participants was relatively small for each subtype. In particular, there were few subjects who had confluent centrilobular emphysema and advanced destructive emphysema, and the 2 subtypes had to be combined for analysis. Second, as we did not externally validate our results, a prospective study with a large population is needed. Third, we classified PSE subtype if substantial PSE was present, regardless of other CT features, and thus our PSE subtype groups may not necessarily reflect the characteristics of pure PSE. Fourth, in our study, COPD were defined according to the chronic exposure to noxious agents, chronic respiratory symptoms, postbronchodilator ratio of $\mathrm{FEV} 1 / \mathrm{FVC}$ of $<0.7$, and no serious structural change. Smoking is a significant factor that causes COPD, but various factors other than smoking cause COPD. Our study included patients with non-smoker COPD, so there may be limitation in generalizing smoker COPD. Therefore, further validation is needed.

In conclusion, we demonstrate that visual evaluation of CT images can provide information on lung structure changes and COPD subtypes that cannot currently be identified by quantitative CT. The PSE subtype had better lung function than the severe centrilobular emphysema subtype, and had higher value of inflammatory markers, including neutrophil counts and IL-6 levels. Small airway disease occurs early in this disease, before the development of significant emphysema. Thus, CT-based, visually defined subtypes of COPD can be useful for distinguishing the heterogeneity and pathological processes of COPD.

\section{Acknowledgments}

We would like to thank Editage (www.editage.co.kr) for English language editing.

Funding: This research was supported by the Basic Science Research Program through the National Research Foundation of Korea (NRF) funded by the Ministry of Education (NRF 2018R1D1A1B07049670).

\section{Footnote}

Reporting Checklist: The authors have completed the STROBE reporting checklist. Available at http://dx.doi. org/10.21037/jtd-20-3041

Data Sharing Statement: Available at http://dx.doi. org/10.21037/jtd-20-3041

Peer Review File: Available at http://dx.doi.org/10.21037/jtd20-3041

Conflicts of Interest: All authors have completed the ICMJE uniform disclosure form (available at http://dx.doi. org/10.21037/jtd-20-3041). The authors have no conflicts of interest to declare.

Ethical Statement: The authors are accountable for all aspects of the work in ensuring that questions related to the accuracy or integrity of any part of the work are appropriately investigated and resolved. The study was conducted in accordance with the Declaration of Helsinki (as revised in 2013). This study was approved by the institutional review board of Kangwon National University hospital (approval \#2012-06-007), and all participants provided written informed consent.

Open Access Statement: This is an Open Access article distributed in accordance with the Creative Commons Attribution-NonCommercial-NoDerivs 4.0 International License (CC BY-NC-ND 4.0), which permits the noncommercial replication and distribution of the article with the strict proviso that no changes or edits are made and the 
original work is properly cited (including links to both the formal publication through the relevant DOI and the license). See: https://creativecommons.org/licenses/by-nc-nd/4.0/.

\section{References}

1. Roca J, Vargas C, Cano I, et al. Chronic Obstructive Pulmonary Disease heterogeneity: challenges for health risk assessment, stratification and management. J Transl Med 2014;12 Suppl 2:S3.

2. Park J, Hobbs BD, Crapo JD, et al. Subtyping COPD by Using Visual and Quantitative CT Imaging Features. Chest 2020;157:47-60.

3. Lowe KE, Regan EA, Anzueto A, et al. COPDGene(®) 2019: Redefining the Diagnosis of Chronic Obstructive Pulmonary Disease. Chronic Obstr Pulm Dis 2019;6:384-99.

4. Koo HJ, Lee SM, Seo JB, et al. Prediction of Pulmonary Function in Patients with Chronic Obstructive Pulmonary Disease: Correlation with Quantitative CT Parameters. Korean J Radiol 2019;20:683-92.

5. Schroeder JD, McKenzie AS, Zach JA, et al. Relationships Between Airflow Obstruction and Quantitative CT Measurements of Emphysema, Air Trapping, and Airways in Subjects With and Without Chronic Obstructive Pulmonary Disease. AJR Am J Roentgenol 2013;201:W460-70.

6. Gevenois PA, de Maertelaer V, De Vuyst P, et al. Comparison of computed density and macroscopic morphometry in pulmonary emphysema. Am J Respir Crit Care Med 1995;152:653-7.

7. Madani A, De Maertelaer V, Zanen J, et al. Pulmonary emphysema: radiation dose and section thickness at multidetector CT quantification--comparison with macroscopic and microscopic morphometry. Radiology 2007;243:250-7.

8. Lynch DA, Austin JHM, Hogg JC, et al. CT-Definable Subtypes of Chronic Obstructive Pulmonary Disease: A Statement of the Fleischner Society. Radiology 2015;277:192-205.

9. Lynch DA, Moore CM, Wilson C, et al. CT-based Visual Classification of Emphysema: Association with Mortality in the COPDGene Study. Radiology 2018;288:859-66.

10. Lee H, Hong Y, Lim MN, et al. Inflammatory biomarkers and radiologic measurements in never-smokers with COPD: A cross-sectional study from the CODA cohort. Chron Respir Dis 2018;15:138-45.

11. Vogelmeier CF, Criner GJ, Martinez FJ, et al. Global
Strategy for the Diagnosis, Management, and Prevention of Chronic Obstructive Lung Disease 2017 Report. GOLD Executive Summary. Am J Respir Crit Care Med 2017;195:557-82.

12. Miller MR, Hankinson J, Brusasco V, et al. Standardisation of spirometry. Eur Respir J 2005;26:319-38.

13. Galbán CJ, Han MK, Boes JL, et al. Computed tomography-based biomarker provides unique signature for diagnosis of COPD phenotypes and disease progression. Nat Med 2012;18:1711-5.

14. Bhatt SP, Washko GR, Hoffman EA, et al. Imaging Advances in Chronic Obstructive Pulmonary Disease. Insights from the Genetic Epidemiology of Chronic Obstructive Pulmonary Disease (COPDGene) Study. Am J Respir Crit Care Med 2019;199:286-301.

15. Lange P, Halpin DM, O'Donnell DE, et al. Diagnosis, assessment, and phenotyping of COPD: beyond FEV . Int J Chron Obstruct Pulmon Dis 2016;11 Spec Iss:3-12.

16. Pompe E, Strand M, van Rikxoort EM, et al. Five-year Progression of Emphysema and Air Trapping at CT in Smokers with and Those without Chronic Obstructive Pulmonary Disease: Results from the COPDGene Study. Radiology 2020;295:218-26.

17. Mascalchi M, Camiciottoli G, Diciotti S. Lung densitometry: why, how and when. J Thorac Dis 2017;9:3319-45.

18. Mohamed Hoesein FAA, de Jong PA, Lammers JWJ, et al. Contribution of CT Quantified Emphysema, Air Trapping and Airway Wall Thickness on Pulmonary Function in Male Smokers With and Without COPD. COPD 2014;11:503-9.

19. Pompe E, van Rikxoort EM, Schmidt M, et al. Parametric response mapping adds value to current computed tomography biomarkers in diagnosing chronic obstructive pulmonary disease. Am J Respir Crit Care Med 2015;191:1084-6.

20. Pompe E, Galban CJ, Ross BD, et al. Parametric response mapping on chest computed tomography associates with clinical and functional parameters in chronic obstructive pulmonary disease. Respir Med 2017;123:48-55.

21. Kim SS, Seo JB, Lee HY, et al. Chronic obstructive pulmonary disease: lobe-based visual assessment of volumetric CT by Using standard images--comparison with quantitative $\mathrm{CT}$ and pulmonary function test in the COPDGene study. Radiology 2013;266:626-35.

22. Smith BM, Austin JH, Newell JD Jr, et al. Pulmonary emphysema subtypes on computed tomography: the MESA COPD study. Am J Med 2014;127:94.e7-23. 
23. Tanabe N, Vasilescu DM, Hague CJ, et al. Pathological Comparisons of Paraseptal and Centrilobular Emphysema in COPD. Am J Respir Crit Care Med 2020;202:803-11.

24. Barnes PJ. Inflammatory mechanisms in patients with chronic obstructive pulmonary disease. J Allergy Clin Immunol 2016;138:16-27.

25. Suzuki M, Sze MA, Campbell JD, et al. The cellular and molecular determinants of emphysematous destruction in COPD. Sci Rep 2017;7:9562.

26. Andreeva E, Pokhaznikova M, Lebedev A, et al. Spirometry is not enough to diagnose COPD in epidemiological studies: a follow-up study. NPJ Prim Care Respir Med 2017;27:62.

27. Hoenderdos K, Condliffe A. The neutrophil in chronic obstructive pulmonary disease. Am J Respir Cell Mol Biol 2013;48:531-9.

28. Yousuf A, Brightling CE. Biologic Drugs: A New Target Therapy in COPD? COPD 2018;15:99-107.

29. Ruwanpura SM, McLeod L, Miller A, et al. Interleukin-6

Cite this article as: Kang HS, Bak SH, Oh HY, Lim MN, Cha YK, Yoon HJ, Kim WJ. Computed tomography-based visual assessment of chronic obstructive pulmonary disease: comparison with pulmonary function test and quantitative computed tomography. J Thorac Dis 2021;13(3):1495-1506. doi: 10.21037/jtd-20-3041 promotes pulmonary emphysema associated with apoptosis in mice. Am J Respir Cell Mol Biol 2011;45:720-30.

30. MacNee W, Murchison JT. Small Airway Disease or Emphysema: Which Is More Important in Lung Function and FEV1 Decline? An Old Story with a New Twist. Am J Respir Crit Care Med 2016;194:129-30.

31. Bhatt SP, Soler X, Wang X, et al. Association between Functional Small Airway Disease and FEV1 Decline in Chronic Obstructive Pulmonary Disease. Am J Respir Crit Care Med 2016;194:178-84.

32. McDonough JE, Yuan R, Suzuki M, et al. Small-airway obstruction and emphysema in chronic obstructive pulmonary disease. N Engl J Med 2011;365:1567-75.

33. Ogawa E, Nakano Y, Ohara T, et al. Body mass index in male patients with COPD: correlation with low attenuation areas on CT. Thorax 2009;64:20-5.

34. Mair G, Maclay J, Miller JJ, et al. Airway dimensions in COPD: relationships with clinical variables. Respir Med 2010;104:1683-90. 